\title{
Activity-Dependent Ubiquitination of the AMPA Receptor Subunit GluA2
}

\author{
Marc P. Lussier, Yukiko Nasu-Nishimura, and Katherine W. Roche \\ Receptor Biology Section, National Institute of Neurological Disorders and Stroke, National Institutes of Health, Bethesda, Maryland 20892-3704
}

\begin{abstract}
AMPA receptors (AMPARs) are postsynaptic glutamate-gated ion channels that mediate fast excitatory neurotransmission in the mammalian brain. Synaptic activity modulates the density of synaptic AMPARs, thereby affecting synaptic function, learning, and memory. Consequently, there is intense interest in defining the molecular mechanisms regulating AMPAR trafficking. Protein expression in the postsynaptic density of excitatory synapses is tightly regulated by ubiquitination, a posttranslational modification that dynamically regulates protein trafficking and degradation in response to synaptic activity. In this study, we demonstrate that increasing synaptic activity, via treatment with the $\mathrm{GABA}_{\mathrm{A}}$ receptor antagonist bicuculline, rapidly and robustly induces ubiquitination of the GluA2 AMPAR subunit. Similarly, treatment with AMPAR agonists results in GluA2 ubiquitination, which suggests that ligand binding plays a critical role. Finally, we find that clathrin- and dynamin-dependent endocytosis of AMPARs is required for activity-dependent GluA2 ubiquitination. Our finding that GluA2 undergoes activity-dependent ubiquitination expands our understanding of how ubiquitination regulates synaptic plasticity.
\end{abstract}

\section{Introduction}

Glutamate mediates the majority of excitatory neurotransmission in the mammalian brain, and the regulation of glutamate receptors is critical for synaptic function. Activity-dependent changes in synaptic strength are directly mediated by AMPA-type glutamate receptors (AMPARs). AMPAR subunits (GluA1-4) predominantly assemble as tetrameric receptors formed with GluA1/2 or GluA2 complexes in hippocampal pyramidal cells (Wenthold et al., 1996; Lu et al., 2009), which means GluA2 is present in the vast majority of hippocampal AMPARs. GluA2 critically defines the biophysical properties of AMPARs because GluA2-containing AMPARs are calcium impermeable (S. CullCandy et al., 2006; Isaac et al., 2007).

The density of postsynaptic AMPARs is tightly regulated by lateral diffusion, endocytosis, and exocytosis. Additional intracellular trafficking steps are essential for regulating AMPAR density at synapses. Plasma membrane receptors are internalized through endocytic vesicles that fuse with early endosomes. The membrane-bound receptors are then sorted and trafficked through either recycling endosomes back to the plasma membrane or late endosomes en route to lysosomes. Endocytosed AMPARs can be recycled back to the plasma membrane or shuttled to lysosomes for degradation (Ehlers, 2000; Carroll et al., 2001; Park et al., 2004, 2006; Groc and Choquet, 2006). The

\footnotetext{
Received Nov. 11, 2010; accepted Dec. 27, 2010.

The National Institute of Neurological Disorders and Stroke Intramural Research Program (K.W.R.) supported this research and M.P.L. is the recipient of a postdoctoral fellowship from Le Fond de la Recherche en Santé du Québec. We thank John D. Badger II for technical assistance.

Correspondence should be addressed to Katherine W. Roche, National Institute of Neurological Disorders and Stroke, Building 35, Room 2C-903, 9000 Rockville Pike, Bethesda, MD 20892-3704. E-mail: rochek@ninds.nih.gov.

Y. Nasu-Nishimura's present address: Institute of Molecular and Cellular Biosciences, The University of Tokyo, Tokyo 113 0032, Japan.

DOI:10.1523/JNEUROSCI.5944-10.2011

Copyright $\odot 2011$ the authors $\quad 0270-6474 / 11 / 313077-05 \$ 15.00 / 0$
}

mechanisms underlying AMPAR targeting to either recycling or lysosomal pathways remain poorly understood. Thus the mechanisms underlying AMPAR trafficking and degradation are decisive determinants of synaptic function, learning, and memory.

Ubiquitination, the covalent attachment of the 76 aa ubiquitin to lysine residues within target proteins, is a posttranslational modification that can target proteins for degradation and/or influence the intracellular sorting of receptors. Unlike cytosolic proteins, which are typically degraded by the proteasome, membrane-bound receptor degradation usually occurs in lysosomes (Hicke and Dunn, 2003). Ubiquitination plays an important role in the rapid bidirectional activity-dependent synaptic expression of proteins including SHANK, GKAP, AKAP79/159, and PSD-95 (Colledge et al., 2003; Ehlers, 2003). In addition, ubiquitination is also known to play a role in the lysosomal targeting and degradation of many synaptic and nonsynaptic transmembrane proteins (Ehlers, 2000; Saliba et al., 2007, 2009; Arancibia-Cárcamo et al., 2009; Pryor and Luzio, 2009). However, direct ubiquitination of mammalian AMPA receptors has not been explored.

Here, we demonstrate that the AMPAR subunit GluA2 undergoes ubiquitination after increased synaptic activity or treatment with AMPAR agonists. GluA2 ubiquitination is regulated by calcium and AMPAR ligand binding, and is dependent on endocytosis. Thus, direct modification of AMPARs by ubiquitination can rapidly modify the complement of synaptic receptors in an activity-dependent manner.

\section{Materials and Methods}

Materials. Serum-free Neurobasal media, glutamine, B-27 supplement, HEPES, and BAPTA/AM were purchased from Invitrogen. Pharmacological agents [( $S$ )-AMPA, NMDA, kainate, DHPG, dynasore, and bicuculline methiodide] were purchased from Tocris Bioscience. Protein 


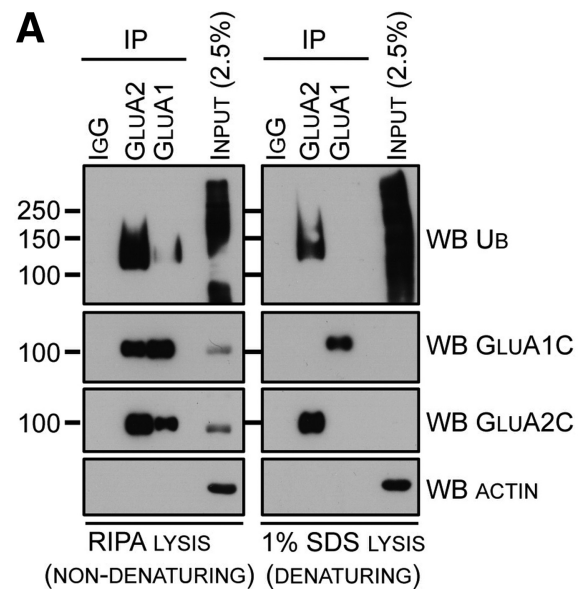

B C

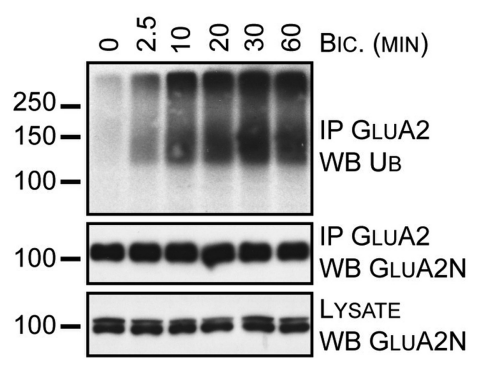

Figure 1. Neuronal activity specifically promotes GluA2 ubiquitination. A, Cortical neurons were incubated in ACSF before bicuculline was added for 15 min. Cells were lysed in RIPA or $1 \%$ SDS as described under Materials and Methods. AMPARs were immunoprecipitated using nonimmune lgG-, GluA1-, or GluA2-specific antibodies, and immunoblotted for ubiquitin, GluA1, GluA2, and actin. B, Cortical neurons were incubated in ACSF before bicuculline was added for the indicated time. Cells were lysed, and GluA2 was immunoprecipitated using an anti-GluA2 antibody, and immunoblotted for ubiquitin. C, Cortical neurons were incubated in the absence (-) or presence of bicuculline for $30 \mathrm{~min}$ at $37^{\circ} \mathrm{C}$ in ACSF. Bicuculline was washed and neurons in fresh ACSF were returned to $37^{\circ} \mathrm{C}$ for recovery periods of 15 or $30 \mathrm{~min}$. Cells were lysed and GluA2 was immunoprecipitated using an anti-GluA2 antibody. Samples were subjected to SDS-PAGE followed by immunoblotted with the indicated antibodies.

A-Sepharose, sucrose, $N$-ethylmaleimide, D-APV, and NBQX were purchased from Sigma-Aldrich.

Antibodies. Quality Controlled Biochemicals generated the affinitypurified rabbit polyclonal antibodies against GluA1C (SHSSGMPLGATGL) and GluA2 (EGYNVYGIESVKI). The GluA2 antibody recognizes mainly GluA2 and to a lesser extent GluA3, as determined by immunoblot (not shown). The affinity-purified rabbit polyclonal GluA2C (VAKNPQNINPSSSQNS) antibody was a kind gift from Robert J. Wenthold (National Institutes of Health, Bethesda, MD). The following antibodies are commercially available: mouse anti-GluA2 6C4 $\mathrm{N}$-terminal antibody (Invitrogen), mouse anti-ubiquitin P4D1 antibody (1:750, Santa Cruz Biotechnology), mouse anti- $\beta$-actin (1:1000, Applied Biological Materials), and peroxidase-conjugated sheep anti-mouse or donkey anti-rabbit secondary antibodies (1:10,000, GE Healthcare).

Primary neuronal cultures. Rat cortical neurons (DIV17-21) were used and prepared from E18 Sprague Dawley rats (Harlan) as previously described (Roche and Huganir, 1995). Neurons were plated at a density of $7.5 \times 10^{6}$ on poly-D-lysine-coated $100 \mathrm{~mm}$ dish and grown in Neurobasal media supplemented with glutamine and B-27.

In vivo ubiquitination and immunoprecipitation. Experiments were conducted in artificial CSF (ACSF, in mM; 10 HEPES, $150 \mathrm{NaCl}, 3 \mathrm{KCl}, 10$ glucose, $2 \mathrm{CaCl}_{2}$, and $1 \mathrm{MgCl}_{2}, \mathrm{pH}$ adjusted with $\mathrm{NaOH}$ to 7.3 ). Note that in Figure $2 C, 2 \mathrm{~mm} \mathrm{CaCl}_{2}$ was replaced by $1 \mathrm{~mm}$ EGTA. Neurons were washed with warm buffer, and the pharmacological agents were added directly to the buffer for a pretreatment of $45 \mathrm{~min}$ with $50 \mu \mathrm{M}$ NBQX, 50 $\mu \mathrm{M}$ D-APV, or $10 \mu \mathrm{M}$ nifedipine; $30 \mathrm{~min}$ with $80 \mu \mathrm{M}$ dynasore; or $2 \mathrm{~min}$ with $450 \mathrm{~mm}$ sucrose. For the BAPTA/AM experiment (see Fig. 2 D), cells were loaded for $1 \mathrm{~h}$ with $50 \mu \mathrm{M}$ BAPTA/AM, then incubated in fresh buffer for an additional $15 \mathrm{~min}$. Following pretreatments, we used $20 \mu \mathrm{M}$ bicuculline (for time period indicated in figure legends) or $100 \mu \mathrm{M}$ AMPA for $10 \mathrm{~min}$. The cells were cooled on ice, washed in ice-cold ACSF containing $10 \mathrm{~mm} N$-ethylmaleimide (NEM), and then lysed in ice-cold RIPA buffer $[150 \mathrm{~mm} \mathrm{NaCl}, 1 \%$ Triton X-100, 0.5\% deoxycholate, $0.1 \%$ SDS, 5 mm EDTA, 20 mm Tris-HCl, pH 8.0, $1 \times$ Complete (Roche), 10 mM NEM]. After centrifugation, the protein content of the supernatant (soluble material) was evaluated using BCA Protein Assay (Thermo Scientific). Three hundred to five hundred micrograms of protein were mixed with $6 \mu \mathrm{g}$ of anti-GluA2 antibody and $30 \mu \mathrm{l}$ of slurry protein A-Sepharose and incubated overnight at $4^{\circ} \mathrm{C}$. For denaturing immunoprecipitation, the above procedure was modified as follows. After stimulation, cells were collected by centrifugation in ACSF containing $10 \mathrm{~mm}$ NEM, sonicated for $5 \mathrm{~s}$ in $100 \mu \mathrm{l}$ of denaturing buffer [1\% SDS, $5 \mathrm{~mm}$ EDTA, 20 mм Tris-HCl, pH 8.0, $1 \times$ Complete (Roche), 10 mm NEM], and then heated at $95^{\circ} \mathrm{C}$ for $7 \mathrm{~min}$. Samples were cooled to room tem- perature and then diluted ( $900 \mu \mathrm{l}$ of ice-cold RIPA buffer without SDS) before being incubated at $4^{\circ} \mathrm{C}$ for $90 \mathrm{~min}$ with agitation. The soluble fraction was cleared by centrifugation and the supernatant used for immunoprecipitation as described above. All immunoprecipitated samples were washed with ice-cold RIPA buffer, eluted with $2 \times$ Laemmli buffer, heated for $5 \mathrm{~min}$ at $95^{\circ} \mathrm{C}$, and separated on $7 \%$ SDS-PAGE. For each immunoprecipitation, 20\% was probed for GluA2 and 80\% for ubiquitin. The immunoprecipitation efficiency was similar between the two lysis conditions tested (Fig. $1 \mathrm{~A}$ ); however, we chose the non-denaturing/ RIPA lysis for all subsequent experiments because very little signal was obtained for AMPARs in the total input under denaturing lysis conditions.

Immunoblotting. Samples were separated by SDS-PAGE and transferred to a $0.45 \mu \mathrm{m}$ PVDF membrane (Millipore). Membranes were blocked in TBS-T (TBS with $0.1 \%$ Tween 20 ) containing $5 \%(w / v)$ nonfat skim milk before incubation with primary antibodies in TBS-T. Peroxidase-conjugated secondary antibodies, diluted in TBS-T, were detected using SuperSignal West Pico Chemiluminescent Substrate (Thermo Scientific) and T2 blue-sensitive films (White Mountain Imaging/Fujifilm). For each study, a representative immunoblot from at least three independent experiments is shown.

\section{Results}

Although many synaptic proteins have been shown to be ubiquitinated (for review, see Mabb and Ehlers, 2010), AMPAR subunits remain conspicuously absent from this list. To determine whether AMPARs are a target for ubiquitination and whether activity can drive this process, we treated cultured cortical neurons with bicuculline to increase neuronal synaptic activity. Immunoprecipitated GluA1 or GluA2 was compared under nondenaturing (RIPA) and denaturing (1\% SDS) lysis conditions (Fig. 1A). Immunoblotting for ubiquitin revealed that GluA2, but not GluA1, is specifically ubiquitinated under the denaturing conditions that completely disrupt GluA1/2 complexes. The ubiquitination of GluA2 under denaturing conditions clearly demonstrates the direct modification of the protein.

Next, we investigated the GluA2 ubiquitination time course by treating cultured neurons with bicuculline for the indicated time periods, followed by immunoprecipitation of GluA2 and immunoblotting for ubiquitin. At the zero time point, very little ubiquitination of GluA2 was present, which suggests that GluA2 ubiquitination is negligible under basal 
A
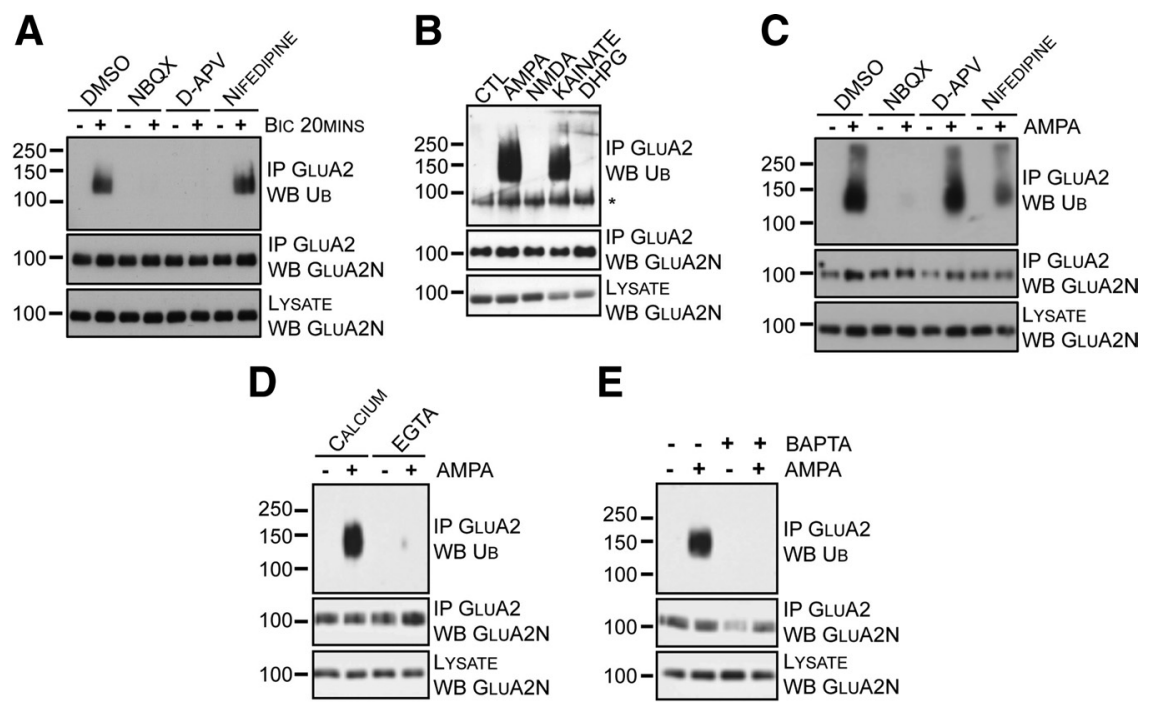

$\mathrm{E}$

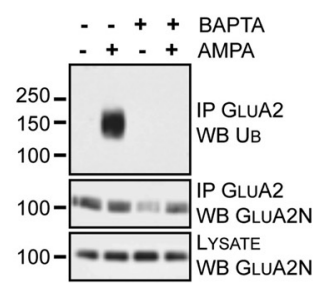

Figure 2. Activity-dependent GluA2 ubiquitination requires AMPAR activation and calcium. $A$, Cortical neurons were incubated at $37^{\circ} \mathrm{C}$ in ACSF for $30 \mathrm{~min}$ with the indicated inhibitors before bicuculline $(+)$ was added for $20 \mathrm{~min}$ at $37^{\circ} \mathrm{C}$. Cells were lysed and GluA2 was immunoprecipitated using an anti-GluA2 antibody. $\boldsymbol{B}$, Cortical neurons were incubated in the absence (CTL) or presence of glutamate receptor agonists (100 $\mu \mathrm{m}$ AMPA, $30 \mu \mathrm{M}$ NMDA, $10 \mu \mathrm{m}$ kainate, or $100 \mu \mathrm{m} \mathrm{DHPG}$ ) for $10 \mathrm{~min}$ in ACSF. Cells were lysed, and GluA2 was immunoprecipitated with an anti-GluA2 antibody. The asterisk (*) indicates a nonspecific band. C, Cortical neurons were incubated at $37^{\circ} \mathrm{C}$ in ACSF for 30 min with the indicated inhibitors before adding (+) or omitting (-) AMPA for 10 min at $37^{\circ} \mathrm{C}$. Cells were lysed, and GluA2 was immunoprecipitated using an anti-GluA2 antibody. $\boldsymbol{D}$, Cortical neurons were incubated in ACSF containing calcium or EGTA in the absence $(-)$ or presence $(+)$ of AMPA for $10 \mathrm{~min}$ at $37^{\circ} \mathrm{C}$. Neurons were lysed, and GluA2 was immunoprecipitated. $\boldsymbol{E}$, Cortical neurons were incubated at $37^{\circ} \mathrm{C}$ in ACSF for $60 \mathrm{~min}$ in the absence $(-)$ or presence $(+)$ of BAPTA/AM. Cells were washed and returned to $37^{\circ} \mathrm{C}$ for $15 \mathrm{~min}$ in fresh ACSF. Neurons were treated without ( - ) or with ( + ) AMPA for $10 \mathrm{~min}$. Cells were lysed, and GluA2 was immunoprecipitated.

conditions (Fig. $1 B$ ). Incubation with bicuculline induced rapid and robust ubiquitination of GluA2, within minutes of bicuculline application (Fig. $1 B$ ).

The reversibility of GluA2 ubiquitination was investigated using a recovery experiment. We treated cultured cortical neurons with bicuculline for $30 \mathrm{~min}$ (Fig. 1C), and then allowed neurons to recover for 15 or $30 \mathrm{~min}$ in drug-free media. Following lysis, GluA2 was immunoprecipitated, and proteins were immunoblotted. We found that the GluA2 ubiquitination was almost completely eliminated after $30 \mathrm{~min}$ of bicuculline withdrawal.

Bicuculline treatment causes both massive epileptiform activity and synaptic release of glutamate; thus, multiple mechanisms could modulate GluA2 ubiquitination, including activation of AMPARs and/or NMDARs. To evaluate the specific mechanism involved in GluA2 ubiquitination, we blocked AMPARs, NMDARs, and voltage-gated calcium channels (VGCCs) with NBQX, D-APV, and nifedipine, respectively. Bicuculline was applied after incubation with these specific inhibitors. Neurons were lysed, GluA2 immunoprecipitated, and immunoblotted for ubiquitin. Blockade of AMPARs or NMDARs inhibited GluA2 ubiquitination; however, blocking VGCCs had no effect on bicuculline-induced GluA2 ubiquitination (Fig. 2A).

To determine which glutamate receptors can trigger GluA2 ubiquitination, we treated neurons with a variety of specific glutamate receptor agonists (AMPA, NMDA, kainate, and DHPG) for $10 \mathrm{~min}$. Following GluA2 immunoprecipitation and ubiquitin immunoblotting, we found that GluA2 ubiquitination was triggered only when AMPARs were selectively activated (AMPA or kainate), but not when neurons were exposed to NMDAR (NMDA) or mGluR (DHPG) agonists (Fig. 2B). This result suggests that ligand binding to AMPARs, leading to channel opening and structural changes (Armstrong and Gouaux, 2000), may be involved in GluA2 ubiquitination.
As we have shown, blockade of NMDARs during synaptic stimulation (Fig. $2 A$ ) and inhibition of VGCCs during the AMPA treatment (Fig. 2C) interfere with GluA2 ubiquitination. We hypothesized that calcium entry might be required for GluA2 ubiquitination, and tested this possibility by comparing our standard calcium-containing ACSF with a modified ACSF solution lacking calcium and containing EGTA. To eliminate any potential confounding effects on glutamate release, we induced GluA2 ubiquitination using AMPA treatment rather than bicuculline. Cultured cortical neurons were incubated in the indicated solutions and treated with AMPA. AMPA treatment effectively induced GluA2 ubiquitination in the calcium-containing ACSF (Fig. 2D); however, the AMPA-triggered GluA2 ubiquitination was inhibited in the absence of extracellular calcium. This finding suggests that calcium influx, following AMPAR activation, is required for GluA2 ubiquitination. To determine whether intracellular calcium is also essential, we loaded the cortical neurons with BAPTA/ AM, a cell-permeable calcium chelator, before adding AMPA. Intracellular calcium chelation also inhibited AMPAinduced GluA2 ubiquitination (Fig. 2E). Together, these results show that calcium is critical for activitydependent GluA2 ubiquitination.

To investigate whether ubiquitination of GluA2 occurs at the plasma membrane or following endocytosis, we treated neurons with dynasore to pharmacologically inhibit the GTPase-activity of dynamin (Macia et al., 2006). Because blocking the GTPase activity of dynamin could also affect vesicular exocytosis (Newton et al., 2006), we also treated neurons with a high concentration of sucrose to inhibit clathrin-coated pit formation. We found that blocking either clathrin- or dynamin-mediated endocytosis inhibited bicuculline-induced GluA2 ubiquitination, whereas treatment with DMSO had no effect (Fig. $3 A$ ). Together, these results indicate that bicuculline-induced GluA2 ubiquitination occurs after endocytosis.

\section{Discussion}

Protein expression at the postsynaptic density (PSD) undergoes dynamic modifications in response to changes in synaptic activity. In a comprehensive study, Ehlers (2003) demonstrated that the $\mathrm{GABA}_{\mathrm{A}}$ receptor antagonist bicuculline induced robust bidirectional changes in the $\mathrm{PSD}$, including regulating the ubiquitination state of a variety of postsynaptic proteins. Therefore, ubiquitination has the potential to sculpt the PSD composition, and consequently modulate the activity and plasticity of synapses (DiAntonio and Hicke, 2004). In the current study, we investigated the role of synaptic activation in regulating AMPAR ubiquitination and internalization. Increasing neuronal activity using bicuculline triggers calcium- and NMDAR-dependent GluA2 ubiquitination. In addition, synaptic activity promotes AMPAR ubiquitination after internalization.

To gain insight into the signaling mechanisms regulating GluA2 ubiquitination, we screened specific agonists known 
to induce AMPAR endocytosis (AMPA, NMDA, kainate, or DHPG), and found that AMPAR agonists specifically triggered GluA2 ubiquitination (AMPA or kainate) (Fig. 2B). These findings suggest that ubiquitination may result from structural changes in AMPARs induced by agonist binding. Indeed, binding of AMPA or kainate in the glutamate-binding cleft causes a conformational change in the structure of GluA2 leading to channel opening (Armstrong and Gouaux, 2000). In addition, the binding of competitive antagonists, such as DNQX, induces minimal structural changes and does not activate the wild-type receptor (Sobolevsky et al., 2009). This may explain why NBQX fails to promote GluA2 ubiquitination by itself (Fig. 2A). In addition to AMPAR ligand binding, GluA2 ubiquitination requires calcium influx. Indeed, AMPAinduced GluA2 ubiquitination is inhibited by extracellular and intracellular calcium chelation (Fig. 2D,E), partially blocked by nifedipine, and surprisingly not inhibited by D-APV application (Fig. 2C). Moreover, bicuculline-induced GluA2 ubiquitination is entirely blocked by NBQX or D-APV (Fig. 2A), and NMDA application is insufficient to promote GluA2 ubiquitination (Fig. 2B) (Colledge et al., 2003), even though NMDAR activation is known to trigger AMPAR endocytosis (Carroll et al., 1999, Zhou et al., 2001). Together these results support a role for conformational changes in AMPAR structure in a calcium-dependent ubiquitination process.

Because inhibitors differentially blocked GluA2 ubiquitination initiated by bicuculline versus AMPA treatments (Fig. $2 A, C$ ), our results suggest that distinct pathways may trigger the necessary calcium influx. Specifically, it is surprising that we find calcium-dependent GluA2 ubiquitination following AMPA treatment because GluA2-containing AMPARs are calcium impermeable (S. Cull-Candy et al., 2006; Isaac et al., 2007). It is possible that AMPA application stimulates both GluA2containing and GluA2-lacking AMPARs, and the resulting calcium influx circumvents the activation of NMDARs, which is normally the trigger for GluA2 ubiquitination following synaptic stimulation. This might also explain the residual AMPAtriggered GluA2 ubiquitination observed following nifedipine pretreatment.

Because AMPAR internalization is dynamin dependent (Carroll et al., 1999), we tested the effect of clathrin- and dynamin-dependent endocytosis on activity-induced GluA2 ubiquitination (Fig. $3 A$ ). Our results show that interfering with endocytosis using dynasore or sucrose treatment inhibited agonist-induced GluA2 ubiquitination, suggesting that endocytosis precedes ubiquitination. Therefore, one possible explanation for the agonist-dependent ubiquitination is an increased GluA2 endocytosis, as opposed to a mechanism directly regulating postendocytic GluA2 ubiquitination. Regardless of the precise mechanisms, our studies demonstrate a physiological role for ubiquitin coupling in GluA2 endocytosis.

The specific function of ubiquitin in the endocytic pathway is difficult to study endogenously, mainly because both the cargo and members of the endocytic machinery are ubiquitinated. It was recently found that multiple monoubiquitinations (multiubiquitination), rather than polyubiquitination, of transmem- brane proteins are sufficient to trigger internalization and subsequent endosomal sorting for recycling or lysosomal degradation (Haglund et al., 2003). In addition, accumulating evidence indicates that the Lys63-polyubiquitin chain is involved in receptor endocytosis (Geetha et al., 2005; Vina-Vilaseca and Sorkin, 2010). Furthermore, since ubiquitination is a highly regulated process that can independently regulate surface expression of plasma membrane receptors and their postendocytic sorting for lysosomal degradation, the ubiquitin coupling to a substrate protein almost certainly depends on a specific subcellular localization of both the ubiquitin E3 ligase and the substrate. In the context of our results, which demonstrate that GluA2 undergoes activity-dependent postendocytosis ubiquitination, one plausible hypothesis involves the action of a yet undefined E3-ligase that acts specifically on internalized GluA2-containing AMPARs to regulate their postendocytic sorting and degradation. Future work should provide evidence for this hypothesis to understand the specific regulation of postendocytic GluA2 ubiquitination, as well as identifying the ubiquitinated lysine(s) on GluA2 and the type of ubiquitin chain conjugated to AMPARs.

Glutamate receptor endocytosis and ubiquitination have been studied in Caenorhabditis elegans (Burbea et al., 2002). Using the unc-11 mutant (ortholog of mammalian clathrin-adaptor protein AP180), it was demonstrated that ubiquitinated and total GFP-tagged GLR-1 protein levels were increased, indicating that unc-11 and GLR-1 ubiquitination act synergistically to regulate glutamate receptor trafficking and degradation in C. elegans. In agreement with this study, our results suggest that activitydependent GluA2 ubiquitination occurs during GluA2 endocytosis (Fig. $3 A$ ). Nonetheless, we cannot rule out the possibility that AMPAR ubiquitination occurs at the plasma membrane, before endocytosis, independent of synaptic activation. The precise mechanism or mechanisms regulating the timing of GluA2 ubiquitination raises interesting questions for future studies.

Based on our results, we propose the following model (Fig. $3 B$ ) for AMPAR trafficking following synaptic activity. First, glutamate is released and binds NMDARs, causing a local calcium influx. In addition, glutamate/agonist binding to AMPARs causes AMPAR channel opening. GluA2-containing AMPARs diffuse away from the postsynaptic density to a perisynaptic site where a clathrin-coated pit is assembled. Following internalization, 
GluA2 is ubiquitinated. Ubiquitinated AMPARs may be trafficked to lysosomes for degradation or deubiquitinated and recycled to the plasma membrane. Our current study expands the role for ubiquitination at excitatory synapses, showing the direct regulation of AMPARs. These findings demonstrate a rapid ubiquitination of AMPARs in response to synaptic activity that targets receptors for internalization. The reversibility of AMPAR ubiquitination illuminates the importance of also studying the dynamics of deubiquitination in future work.

\section{References}

Arancibia-Cárcamo IL, Yuen EY, Muir J, Lumb MJ, Michels G, Saliba RS, Smart TG, Yan Z, Kittler JT, Moss SJ (2009) Ubiquitin-dependent lysosomal targeting of GABA (A) receptors regulates neuronal inhibition. Proc Natl Acad Sci U S A 106:17552-17557.

Armstrong N, Gouaux E (2000) Mechanisms for activation and antagonism of an AMPA-sensitive glutamate receptor: crystal structures of the GluR2 ligand binding core. Neuron 28:165-181.

Burbea M, Dreier L, Dittman JS, Grunwald ME, Kaplan JM (2002) Ubiquitin and AP180 regulate the abundance of GLR-1 glutamate receptors at postsynaptic elements in C. elegans. Neuron 35:107-120.

Carroll RC, Beattie EC, Xia H, Lüscher C, Altschuler Y, Nicoll RA, Malenka RC, von Zastrow M (1999) Dynamin-dependent endocytosis of ionotropic glutamate receptor. Proc Natl Acad Sci U S A 96:14112-14117.

Carroll RC, Beattie EC, von Zastrow M, Malenka RC (2001) Role of AMPA receptor endocytosis in synaptic plasticity. Nat Rev Neurosci 2:315-324.

Colledge M, Snyder EM, Crozier RA, Soderling JA, Jin Y, Langeberg LK, Lu H, Bear MF, Scott JD (2003) Ubiquitination regulates PSD-95 degradation and AMPA receptor surface expression. Neuron 40:595-607.

Cull-Candy S, Kelly L, Farrant M (2006) Regulation of $\mathrm{Ca}^{2+}$-permeable AMPA receptors: synaptic plasticity and beyond. Curr Opin Neurobiol 16:288-297.

DiAntonio A, Hicke L (2004) Ubiquitin-dependent regulation of the synapse. Annu Rev Neurosci 27:223-246.

Ehlers MD (2000) Reinsertion or degradation of AMPA receptors determined by activity-dependent endocytic sorting. Neuron 28:511-525.

Ehlers MD (2003) Activity level controls postsynaptic composition and signaling via the ubiquitin-proteasome system. Nat Neurosci 6:231-242.

Geetha T, Jiang J, Wooten MW (2005) Lysine 63 polyubiquitination of the nerve growth factor receptor TrkA directs internalization and signaling. Mol Cell 20:301-312.

Groc L, Choquet D (2006) AMPA and NMDA glutamate receptor trafficking: multiple roads for reaching and leaving the synapse. Cell Tissue Res 326:423-438.

Haglund K, Sigismund S, Polo S, Szymkiewicz I, Di Fiore PP, Dikic I (2003)
Multiple monoubiquitination of RTKs is sufficient for their endocytosis and degradation. Nat Cell Biol 5:461-466.

Hicke L, Dunn R (2003) Regulation of membrane protein transport by ubiquitin and ubiquitin-binding proteins. Annu Rev Cell Dev Biol 19:141-172.

Isaac JT, Ashby M, McBain CJ (2007) The role of the GluR2 subunit in AMPA receptor function and synaptic plasticity. Neuron 54:859-871.

Lu W, Shi Y, Jackson AC, Bjorgan K, During MJ, Sprengel R, Seeburg PH, Nicoll RA (2009) Subunit composition of synaptic AMPA receptors revealed by a single-cell genetic approach. Neuron 62:254-268.

Mabb AM, Ehlers MD (2010) Ubiquitination in postsynaptic function and plasticity. Annu Rev Cell Dev Biol 26:179-210.

Macia E, Ehrlich M, Massol R, Boucrot E, Brunner C, Kirchhausen T (2006) Dynasore, a cell-permeable inhibitor of dynamin. Dev Cell 10:839-850.

Newton AJ, Kirchhausen T, Murthy VN (2006) Inhibition of dynamin completely blocks compensatory synaptic vesicle endocytosis. Proc Natl Acad Sci U S A 103:17955-17960.

Park M, Penick EC, Edwards JG, Kauer JA, Ehlers MD (2004) Recycling endosomes supply AMPA receptors for LTP. Science 305:1972-1975.

Park M, Salgado JM, Ostroff L, Helton TD, Robinson CG, Harris KM, Ehlers MD (2006) Plasticity-induced growth of dendritic spines by exocytic trafficking from recycling endosomes. Neuron 52:817-830.

Pryor PR, Luzio JP (2009) Delivery of endocytosed membrane proteins to the lysosome. Biochem Biophys Acta 1793:615-624.

Roche KW, Huganir RL (1995) Synaptic expression of the high-affinity kainate receptor subunit KA2 in hippocampal cultures. Neuroscience 69:383-393.

Saliba RS, Michels G, Jacob TC, Pangalos MN, Moss SJ (2007) Activitydependent ubiquitination of $\mathrm{GABA}_{\mathrm{A}}$ receptors regulates their accumulation at synaptic sites. J Neurosci 27:13341-13351.

Saliba RS, Gu Z, Yan Z, Moss SJ (2009) Blocking L-type voltage-gated Ca ${ }^{2+}$ channels with dihydropyridines reduces gamma-aminobutyric acid type $\mathrm{A}$ receptor expression and synaptic inhibition. J Biol Chem 284:32544-32550.

Sobolevsky AI, Rosconi MP, Gouaux E (2009) X-ray stricture, symmetry and mechanism of an AMPA-subtype glutamate receptor. Nature 462:745-756.

Vina-Vilaseca A, Sorkin A (2010) Lysine 63-linked polyubiquitination of the dopamine transporter requires WW3 and WW4 domains of Nedd4-2 and UBE2D ubiquitin-conjugating enzymes. J Biol Chem 285:7645-7656.

Wenthold RJ, Petralia RS, Blahos J II, Niedzielski AS (1996) Evidence for multiple AMPA receptor complexes in hippocampal CA1/CA2 neurons. J Neurosci 16:1982-1989.

Zhou Q, Xiao M, Nicoll RA (2001) Contribution of cytoskeleton to the internalization of AMPA receptors. Proc Natl Acad Sci U S A 98:12611266. 